\title{
An alternative hypothesis testing strategy for secondary phenotype data in case-control genetic association studies
}

\author{
Sharon M. Lutz ${ }^{1}{ }^{*}$, John E. Hokanson ${ }^{2}$ and Christoph Lange ${ }^{3,4,5,6}$ \\ 1 Department of Biostatistics, University of Colorado, Aurora, CO, USA \\ ${ }^{2}$ Department of Epidemiology, University of Colorado, Aurora, CO, USA \\ ${ }^{3}$ Department of Biostatistics, Harvard School of Public Health, Boston, MA, USA \\ ${ }^{4}$ Channing Laboratory, Harvard Medical School, Boston, MA, USA \\ ${ }^{5}$ Institute for Genomic Mathematics, University of Bonn, Bonn, Germany \\ ${ }^{6}$ German Center for Neurodegenerative Diseases (DZNE), Bonn, Germany
}

\section{Edited by:}

Nathan Morris, Case Western

Reserve University, USA

\section{Reviewed by:}

Qing Lu, Michigan State University, USA

Geetha Chittoor, University of North Carolina at Chapel Hill, USA

*Correspondence:

Sharon M. Lutz, Department of

Biostatistics, University of Colorado,

13001 E. 17th Place, B119 Bldg. 500,

W3128, Aurora, CO 80045, USA

e-mail: sharon.lutz@ucdenver.edu
Motivated by the challenges associated with accounting for the ascertainment when analyzing secondary phenotypes that are correlated with case-control status, Lin and Zeng have proposed a method that properly reflects the case-control sampling (Lin and Zeng, 2009). The Lin and Zeng method has the advantage of accurately estimating effect sizes for secondary phenotypes that are normally distributed or dichotomous. This method can be computationally intensive in practice under the null hypothesis when the likelihood surface that needs to be maximized can be relatively flat. We propose an extension of the Lin and Zeng method for hypothesis testing that uses proportional odds logistic regression to circumvent these computational issues. Through simulation studies, we compare the power and type-1 error rate of our method to standard approaches and Lin and Zeng's approach.

Keywords: secondary phenotype, case-control study, ascertainment, genetic association, proportional odds logistic regression

\section{INTRODUCTION}

For the analysis of secondary phenotype data collected in a case-control study, Lin and Zeng have proposed a method that properly reflects the case-control sampling (Lin and Zeng, 2009). This work is motivated by the challenges associated with accounting for the ascertainment when analyzing secondary phenotypes that are correlated with case-control status. Several methods have been proposed that accurately estimate the odds ratio of genetic variants for binary secondary phenotypes associated with case-control status, but most of these methods do not readily accommodate continuous secondary phenotypes (Greenland, 2003; Kraft, 2007; Richardson et al., 2007; Monsees et al., 2009; Li et al., 2010; Wang and Shete, 2011a,b; He et al., 2012; Li and Gail, 2012). While two of these methods use an inverse probability weighted (IPW) regression approach that can accommodate continuous secondary phenotypes, these methods focus on correcting for the bias in the estimator due to the ascertainment conditions and involve a known disease rate (Richardson et al., 2007; Monsees et al., 2009). Since this paper focuses on hypothesis testing versus estimation of disease-association parameters with an equal number of cases and controls, we do not present these methods here.

Alternatively, the Lin and Zeng method has the advantage of accurately estimating effect sizes for secondary phenotypes that are normally distributed or dichotomous (Lin and Zeng, 2009). Under the null hypothesis when the likelihood surface that needs to be maximized can be relatively flat, this method can be computationally intensive in practice. To circumvent these computational issues, we propose an extension of the Lin and
Zeng method for hypothesis testing that uses proportional odds logistic regression. Since the approach by Lin and Zeng has the advantage that effect sizes can also be estimated, we recommend the following work-flow for the analysis of continuous secondary phenotypes.

1. Test all SNPs with our approach using proportional odds logistic regression since the vast majority of SNPs will be under the null hypothesis.

2. For the significant SNPs, apply Lin and Zeng's method to obtain parameter estimates and confidence intervals.

This proposed approach circumvents the computational issues encountered in the Lin and Zeng approach under the null hypothesis, but utilizes the Lin and Zeng's method to accurately estimate effect sizes for significant SNPs found in Step 1. Through simulation studies, we compare the power and type- 1 error rate of our method to standard approaches and Lin and Zeng's approach.

\section{METHODS}

When the secondary phenotype is normally distributed, Lin and Zeng propose an adjusted score test that incorporates genetic associations with affection status into the test statistic and models the likelihood function as follows (Lin and Zeng, 2009):

$$
\begin{aligned}
\prod_{i=1}^{n} P\left(Y_{i}, X_{i} \mid D_{i}\right)= & \prod_{i=1}^{n}\left\{\frac{P\left(D_{i}=1 \mid X_{i}, Y_{i}\right) P\left(Y_{i} \mid X_{i}\right) P\left(X_{i}\right)}{P\left(D_{i}=1\right)}\right\}^{D_{i}} \\
& \left\{\frac{P\left(D_{i}=0 \mid X_{i}, Y_{i}\right) P\left(Y_{i} \mid X_{i}\right) P\left(X_{i}\right)}{P\left(D_{i}=0\right)}\right\}^{1-D_{i}}
\end{aligned}
$$


where $D$ denotes the case-control status $(1=$ case and $0=$ control), $\mathrm{Y}$ denotes the secondary phenotype, $n$ denotes the total number of subjects, and $\mathrm{X}$ denotes the genotype of interest.

Lin and Zeng calculate $P\left(D_{i}=1\right)=\sum_{y} \sum_{x} P\left(D_{i}=\right.$ $1 \mid x, y) P(y \mid x) P(x)$. The probability $P(D \mid X, Y)$ is defined as a logistic regression model. They model $P(Y \mid X)$ as a logistic regression for dichotomous $Y$ or a linear regression for normally distributed Y. They maximize the likelihood with respect to $P(X)$ by the Newton Raphson algorithm. In this framework, likelihood based statistics (i.e., Wald, score, and likelihood-ratio statistics) can be used to make inference.

The Lin and Zeng approach requires the secondary phenotype to be normally distributed and the method can be problematic under the null hypothesis since the likelihood surface that needs to be maximized can be relatively flat. Since Lin and Zeng's method estimates the parameters in the model by maximizing the likelihood given in Equation (1), the approach is numerically exhaustive when testing a large number of SNPs where a majority of the SNPs are under the null hypothesis. This is a result of the maximization of the likelihood function being difficult under the null hypothesis, since the surface can be flat due to the ascertainment condition.

If the primary goal of the secondary phenotype analysis is hypothesis testing as opposed to estimation of disease-association parameters, an alternative approach is to use the following likelihood composition, which ultimately does not require maximizing a relatively flat likelihood surface. Therefore, for the association testing of secondary phenotypes in case-control studies, we propose using a simpler break down of the likelihood that requires few assumptions.

$$
\prod_{i=1}^{n} P\left(Y_{i}, X_{i} \mid D_{i}\right)=\prod_{i=1}^{n} P\left(X_{i} \mid Y_{i}, D_{i}\right) P\left(Y_{i} \mid D_{i}\right)
$$

Under the null hypothesis, $\mathrm{X}$ is independent of $\mathrm{Y}$ given $\mathrm{D}$ and any confounders. The likelihood ratio test becomes

$$
\begin{aligned}
L R T & =-2 \ln \left(\frac{\prod_{i=1}^{n} P\left(X_{i} \mid D_{i}\right) P\left(Y_{i} \mid D_{i}\right)}{\prod_{i=1}^{n} P\left(X_{i} \mid Y_{i}, D_{i}\right) P\left(Y_{i} \mid D_{i}\right)}\right) \\
& =-2 \ln \left(\frac{\prod_{i=1}^{n} P\left(X_{i} \mid D_{i}\right)}{\prod_{i=1}^{n} P\left(X_{i} \mid Y_{i} D_{i}\right)}\right) \sim \chi_{1 d f}^{2}
\end{aligned}
$$

As a result, one only needs to model $P(X \mid D)$ and $P(X \mid Y, D)$. For an additive genetic model, i.e., $X=0,1,2$, corresponding to allele counts, instead of modeling the likelihood function, one can use a cumulative logistic regression model with proportional odds proportional for $P(X \mid D)$ and the $P(X \mid Y, D)$ such that

$$
\begin{gathered}
\operatorname{logit}[P(X \leq j \mid Y, D)]=\alpha_{1 j}+\delta_{1 Y} Y+\delta_{1 D} D \\
\operatorname{logit}[P(X \leq j \mid D)]=\alpha_{0 j}+\delta_{0 D} D
\end{gathered}
$$

for $j=0,1$. To control for any known confounders, these covariates can be added to Equation (4). This model assumes the same effect for different cumulative logits (Agresti, 2002). If assumptions are not met then we recommend a link function for which the response curve is non-symmetric or adding a dispersion parameter. For imputed dosages, $j$ becomes the number of dosage levels minus one, meaning the levels of $\mathrm{X}$ in the cumulative logistic regression are increased to the number of dosage levels minus one.

\section{SIMULATIONS}

To assess the performance of this approach and compare it to Lin and Zeng's method, we conducted simulation studies following Lin and Zeng's manuscript with a MAF of 0.3 , an additive mode of inheritance, and $\alpha=0.01$ level of significance (Lin and Zeng, 2009). We also compared both of these methods to the standard case-only method, control only method and combined case and control method where both cases and controls are included in the analysis. For the model of the secondary quantitative trait $Y$ and the disease $D$,

$$
\begin{gathered}
Y \mid X \sim N\left(\beta_{0}+\beta_{1} X, \sigma^{2}\right) \\
P(D=1 \mid X, Y)=\frac{\exp \left(\gamma_{0}+\gamma_{1} X+\gamma_{2} Y\right)}{1+\exp \left(\gamma_{0}+\gamma_{1} X+\gamma_{2} Y\right)}
\end{gathered}
$$

where $\beta_{0}=\sigma^{2}=1, \beta_{1}=0$ under the null hypothesis and $\beta_{1}=$ -0.12 under the alternative hypothesis. We let $\gamma_{2}=\log (2), \gamma_{1}$ varies from 0 to $\log (1.5)$, and $\gamma_{0}$ was chosen such that the disease rate is $1 \%$ or $5 \%$. For each combination of simulation parameters, we generated 1000 data sets with 500 cases and 500 controls.

Figure 1 shows the type 1 error rates and power for a disease rate of $1 \%$ and $5 \%$. Our method, using the proportional odds logistic regression, maintains the type 1 error rate and has slightly higher power as compared to Lin and Zeng's method and superior power compared to the other methods. While the proposed method and Lin and Zeng's method have similar power, the proposed method is computationally more feasible under the null hypothesis than Lin and Zeng's method since it does not involve maximizing a relatively flat likelihood surface. The computing time for the proposed approach is under $1 \mathrm{~s}$ per SNP where as the software associated with the Lin and Zeng approach needs to be run multiple times if there are issues with convergence which can take $5 \mathrm{~min}$ to an hour per SNP. When running a GWAS with about 500,000 SNPs, this difference in computing time per SNP can be substantial. To examine this concept further, the plot on the left in Figure 2 shows the log Likelihood specified by Lin and Zeng for varying values of $\beta_{0}$ and $\beta_{1}$ with all other parameters fixed at their true values and for data generated under the null hypothesis with $\gamma_{1}=\log (1.5)$ and the disease rate equal $5 \%$. The plot on the right is the log Likelihood specified by Lin and Zeng for varying values of $\gamma_{1}$ and $\gamma_{2}$ with all other parameters fixed at their true values, and for data generated under the null hypothesis with $\gamma_{1}=\log (1.5)$ and the disease rate equals $5 \%$. The red dots on the 


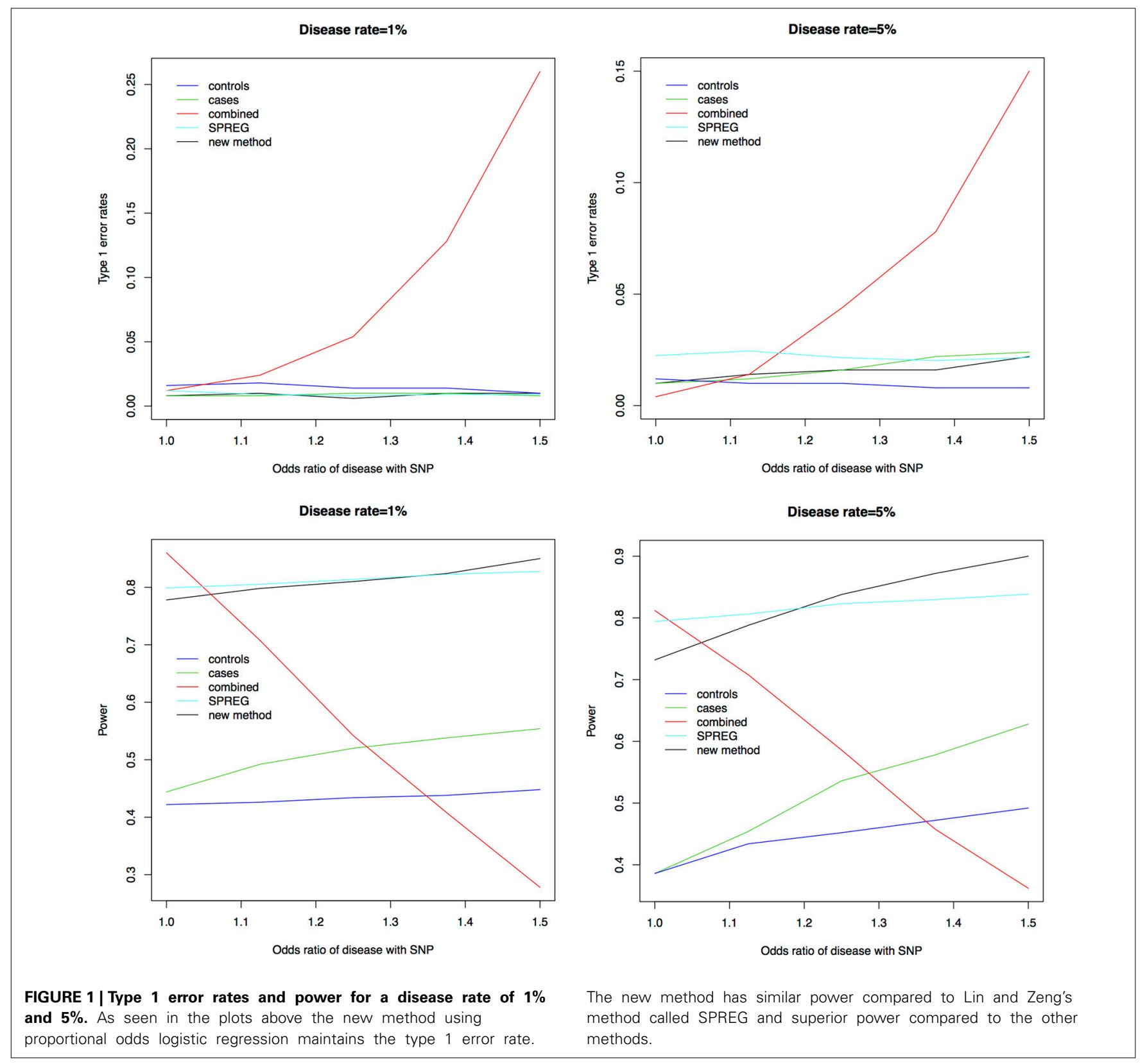

plots represent the true maximum. The surface for $\beta_{0}$ and $\beta_{1}$ has a clear maximum whereas the surface for $\gamma_{1}$ and $\gamma_{0}$ is relatively flat, demonstrating the difficulty in maximizing the likelihood surface defined by Lin and Zeng under the null hypothesis.

\section{DISCUSSION}

While the power of the proposed method is comparable to the method of Lin and Zeng, the proposed approach does not have the issue of maximizing a flat likelihood surface under the hull hypothesis that can be computationally intensive. Since the proposed approach is limited in it's ability to accurately estimate effect sizes while the approach by Lin and Zeng has the advantage that effect sizes can be accurately estimated, we recommend the following work-flow for the analysis of secondary phenotypes.
1. Test all SNPs with the proposed approach using proportional odds logistic regression since the vast majority of SNPs will be under the null hypothesis.

2. For the significant SNPs, apply Lin and Zeng's method to obtain parameter estimates and confidence intervals.

By using our approach to test all the SNPs in the GWAS, the hypothesis testing can be done quickly and efficiently since our approach does not suffer from this issue of maximizing a flat likelihood surface under the null hypothesis. By obtaining parameter estimates for only the significant SNPs with Lin and Zeng's method, one can make sure that the likelihood is properly maximized which is too computational exhaustive to apply to the entire GWAS. 


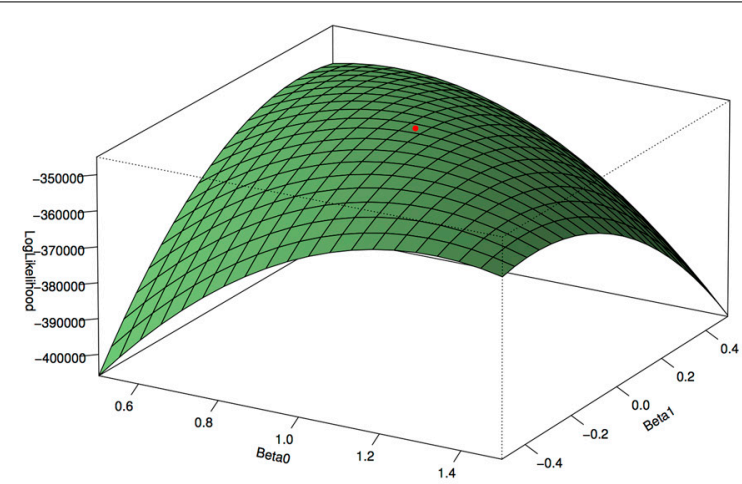

FIGURE 2 | Log Likelihood surface specified by Lin and Zeng. The plot on the left is the log Likelihood specified by Lin and Zeng for varying values of $\beta_{0}$ and $\beta_{1}$ with all other parameters fixed at their true values and for data generated under the null hypothesis with $\gamma_{1}=\log (1.5)$ and the disease rate equal $5 \%$. The plot on the right is the log Likelihood specified by Lin and Zeng for varying values of $\gamma_{1}$ and $\gamma_{2}$ with all other parameters fixed at their true

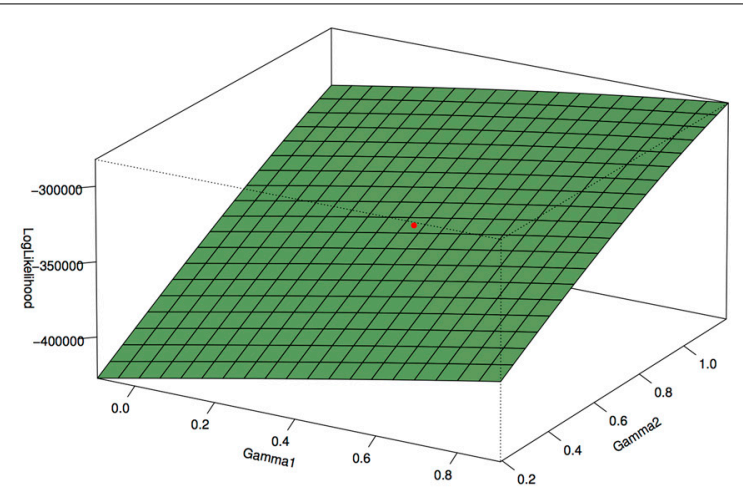

values and for data generated under the null hypothesis with $\gamma_{1}=\log (1.5)$ and the disease rate equal $5 \%$. The red dots on the plots represent the true maximum. The surface for $\beta_{0}$ and $\beta_{1}$ has a clear maximum whereas the surface for $\gamma_{1}$ and $\gamma_{0}$ is relatively flat, demonstrating the difficulty in maximizing the likelihood surface defined by Lin and Zeng under the null hypothesis.
There are potential limitations associated with this strategy of combining two methodological approaches to reduce the computational burden while still being able to estimate the parameters of interest. While the two approaches have comparable power, a relatively small number of SNPs that are significant from the new approach may not be significant in the Lin and Zeng's method and vice versa. Also both approaches may have issues if the case control status is extremely correlated with the secondary phenotype. In this case, the secondary phenotype is not providing new information compared to the case-control status and these methods for testing secondary phenotypes in case-control genetic association studies are not applicable.

\section{ACKNOWLEDGMENTS}

This work was funded by NIH/NHLBI U01 HL089856 Edwin K. Silverman, PI. COPDGene is supported by NHLBI Grant Nos U01HL089897 and U01H1089856.

\section{REFERENCES}

Agresti, A. (2002). Categorical Data Analysis. Hoboken, NJ: Wiley Series in Probability and Statistic. doi: 10.1002/0471249688

Greenland, S. (2003). Quantifying biases in causal models: classical confounding vs collider-stratification bias. Epidemiology 14, 300-306. doi: 10.1097/00001648200305000-00009

He, J., Li, H., Edmondson, A. C., Rader, D. J., and Li, M. (2012). A Gaussian copula approach for the analysis of secondary phenotypes in case control genetic association studies. Biostatistics 3, 497-508. doi: 10.1093/biostatistics/kxr025

Kraft, P. (2007). Letter to the editor: analyses of genome-wide association scans for additional outcomes. Epidemiology 18, 838. doi: 10.1097/EDE.0b013e318154c7e2

Li, H., and Gail, M. H. (2012). Efficient adaptively weighted analysis of secondary phenotypes in case-control genome-wide association studies. Hum. Hered. 73, 159-173. doi: 10.1159/000338943
Li, H., Gail, M. H., Berndt, S., and Chatterjee, N. (2010). Using cases to strengthen inference on the association between single nucleotide polymorphisms and a secondary phenotype in genome-wide association studies. Genet. Epidemiol. 34, 427-433. doi: 10.1002/gepi.20495

Lin, D. Y., and Zeng, D. (2009). Proper analysis of secondary phenotype data in case-control association studies. J. Genet. Epidemiol. 33, 256-265. doi: 10.1002/gepi.20377

Monsees, G. M., Tamimi, R. M., and Kraft, P. (2009). Genome-wide association scans for secondary traits using case-control samples. Genet. Epidemiol. 33, 717-728. doi: 10.1002/gepi.20424

Richardson, D. B., Rzehak, P., Klenk, J., and Weiland, S. K. (2007). Analyses of case control data for additional outcomes. Epidemiology 18, 441-445. doi: 10.1097/EDE.0b013e318060d25c

Wang, J., and Shete, S. (2011a). Power and type I error results for a bias-correction approach recently shown to provide accurate odds ratios of genetic variants for the secondary phenotypes associated with primary diseases. Genet. Epidemiol. 35, 739-743. doi: 10.1002/gepi.20568

Wang, J., and Shete, S. (2011b). Estimation of odds ratios of genetic variants for the secondary phenotypes associated with primary diseases. Genet. Epidemiol. 35, 190-200. doi: 10.1002/gepi.20611

Conflict of Interest Statement: The authors declare that the research was conducted in the absence of any commercial or financial relationships that could be construed as a potential conflict of interest.

Received: 01 April 2014; accepted: 04 June 2014; published online: 01 July 2014. Citation: Lutz SM, Hokanson JE and Lange C (2014) An alternative hypothesis testing strategy for secondary phenotype data in case-control genetic association studies. Front. Genet. 5:188. doi: 10.3389/fgene.2014.00188

This article was submitted to Applied Genetic Epidemiology, a section of the journal Frontiers in Genetics.

Copyright (c) 2014 Lutz, Hokanson and Lange. This is an open-access article distributed under the terms of the Creative Commons Attribution License (CC BY). The use, distribution or reproduction in other forums is permitted, provided the original author(s) or licensor are credited and that the original publication in this journal is cited, in accordance with accepted academic practice. No use, distribution or reproduction is permitted which does not comply with these terms. 